\title{
Time evolution of Wouthuysen-Field coupling
}

\author{
Ishani Roy ${ }^{1}$, Wen $\mathrm{Xu}^{2}$, Jing-Mei $\mathrm{Qiu}^{3}$, Chi-Wang Shu ${ }^{1}$, and Li-Zhi Fang ${ }^{2}$
}

\begin{abstract}
We study the Wouthuysen-Field coupling at early universe with numerical solutions of the integrodifferential equation describing the kinetics of photons undergoing resonant scattering. The numerical solver is developed based on the weighted essentially non-oscillatory (WENO) scheme for the Boltzmann-like integrodifferential equation. This method has perfectly passed the tests of analytic solution and conservation property of the resonant scattering equation. We focus on the time evolution of the Wouthuysen-Field (W-F) coupling in relation to the $21 \mathrm{~cm}$ emission and absorption at the epoch of reionization. We especially pay attention to the formation of the local Boltzmann distribution, $e^{-\left(\nu-\nu_{0}\right) / k T}$, of photon frequency spectrum around resonant frequency $\nu_{0}$ within width $\nu_{l}$, i.e. $\left|\nu-\nu_{0}\right| \leq \nu_{l}$. We show that a local Boltzmann distribution will be formed if photons with frequency $\sim \nu_{0}$ have undergone a ten thousand or more times of scattering, which corresponds to the order of $10^{3}$ yrs for neutral hydrogen density of the concordance $\Lambda \mathrm{CDM}$ model. The time evolution of the shape and width of the local Boltzmann distribution actually doesn't dependent on the details of atomic recoil, photon sources, or initial conditions very much. However, the intensity of photon flux at the local Boltzmann distribution is substantially time-dependent. The time scale of approaching the saturated intensity can be as long as $10^{5}-10^{6}$ yrs for typical parameters of the $\Lambda \mathrm{CDM}$ model. The intensity of the local Boltzmann distribution at time less than $10^{5}$ yrs is significantly lower than that of the saturation state. Therefore, it may not be always reasonable to assume that the deviation of the spin temperature of $21 \mathrm{~cm}$ energy states from cosmic background temperature is mainly due to the W-F coupling if first stars or their emission/absorption regions evolved with a time scale equal to or less than Myrs.
\end{abstract}

Subject headings: cosmology: theory - intergalactic medium - radiation transfer - scattering

\footnotetext{
${ }^{1}$ Division of Applied Mathematics, Brown University, Providence, RI 02912

${ }^{2}$ Department of Physics, University of Arizona, Tucson, AZ 85721

${ }^{3}$ Department of Mathematical and Computer Science, Colorado School of Mines, Golden, CO 80401
} 


\section{Introduction}

It is generally believed that the physical state of the universe at the epoch of reionization can be probed by detecting the redshifted $21 \mathrm{~cm}$ signals from the ionized and heated regions around the first generation of stars (e.g. Furlanetto, Oh, \& Briggs 2006). The emission and absorption of $21 \mathrm{~cm}$ are caused by the deviation of the spin temperature $T_{s}$ of neutral hydrogen from the temperature of cosmic microwave background (CMB) $T_{\mathrm{CMB}}$ at the considered redshift z. Many calculations have been done on the $21 \mathrm{~cm}$ emission and absorption from ionized halos of the first stars (Chuzhoy et al. 2006; Cen 2006; Liu et al. 2007). A common assumption of these calculations is that the deviations of $T_{s}$ from $T_{\mathrm{CMB}}$ are mainly due to the Wouthuysen-Field (W-F) coupling (Wouthuysen, 1952; Field, 1958, 1959). That is, the resonant scattering of Ly $\alpha$ photons with neutral hydrogen atoms locks the color temperature $T_{c}$ of the photon spectrum around the Ly $\alpha$ frequency to be equal to the kinetic temperature of hydrogen gas $T$. Consequently, the spin degree of freedom is determined by the kinetic temperature of hydrogen gas $T$.

The W-F coupling is from the kinetics of photons undergoing resonant scattering, which is described by a Boltzmann integrodifferential equation. All the above-mentioned calculations are based on time-independent solution of the resonant scattering kinetic equation with Fokker-Planck approximation (Chen \& Miralda-Escude, 2004; Hirata, 2006; Furlanetto \& Pritchard 2006; Chuzhoy \& Shapiro 2006). This is equal to assume that the time scale of the onset of the W-F coupling is less than all time scales related to the $21 \mathrm{~cm}$ emission/absorption. However, even in the first paper of the W-F coupling, the problem of time scale has been addressed as follows: "One can infer from this fact that the photons (in a box), after an infinite number of scattering processes on gas atoms with kinetic temperature $T$, will obtain a statistical distribution over the spectrum proportional to the Planck-radiation spectrum of temperature $T$. After a finite but large number of scattering processes, the Planck shape will be produced in a region around the initial frequency" (Wouthuysen, 1952). That is, the W-F coupling is onset only "after a finite but large number of scattering". One cannot assume that the time-independent solution is available for the W-F coupling if the time-scales of the evolution of the first stars and their emission/absorption regions are short. A study on the time evolution of radiation spectrum due to resonant scattering is necessary.

This problem is especially important for the $21 \mathrm{~cm}$ signal from the first stars, as the life times of the first stars are short. The ionized and heated regions around the first stars are strongly time-dependent (Cen 2006; Liu et al. 2007). The $21 \mathrm{~cm}$ emission/absortion regions are located in a narrow shell just outside the ionized region. On the other hand, the speed of

the ionization-front (I-front) is rather high, even comparable to the speed of light. The time scales of the formation and the evolution of the $21 \mathrm{~cm}$ regions can be estimated by $\sim d / c$, 
$d$ being the thickness of the shells of $21 \mathrm{~cm}$ emission and absorption. The time-independent solution would be proper only if $\operatorname{Ly} \alpha$ photons approach the time-independent state in a time shorter than that of the $21 \mathrm{~cm}$ region evolution.

Very few works have been done on the time dependent behavior of the W-F coupling. There is the lack of a time dependent solution even for the Fokker-Planck approximation. The existed time-dependent solvers (e.g. Meiksin, 2006) cannot pass the tests of analytical solutions (Field, 1959). On the other hand, the WENO algorithm is found to be effective to solve the Boltzmann equation (Carrillo et al. 2003) and radiative transfer (Qiu et al. 2006, 2007, 2008). In this paper, we will study the time-dependent behavior of the W-F coupling with the WENO method by numerically solving the integrodifferential equation. In this context, we also develop a numerical solver in accordance with the term of resonant scattering.

The paper is organized as follows. Section 2 presents the basic equations of the resonant scattering of photons. Section 3 very briefly mentions the numerical solver of the WENO scheme and its test with Field's analytic solutions, leaving the details of algorithmic issues to the Appendix. Section 4 presents the time-dependent W-F coupling with a static background. Section 5 shows the numerical results of the W-F coupling in an expanding background. Finally, conclusions are given in Section 6, in which the application to the 21 $\mathrm{cm}$ problem is also addressed.

\section{Basic equations}

\subsection{Radiative transfer equations with resonant scattering}

Since we focus on the time-scales of the W-F coupling, we consider a homogeneous and isotropically expanding infinite medium consisting of neutral hydrogen with temperature $T$. The kinetics of photons in the frequency space is described by the radiative transfer equation with resonant scattering (Hummer \& Rybicki, 1992; Rybicki \& Dell'antonio 1994)

$$
\begin{aligned}
& \frac{\partial J(x, t)}{\partial t}+2 H J(x, t)-\frac{c H}{v_{T}} \frac{\partial J(x, t)}{\partial x}= \\
& -k c \phi(x) J(x, t)+k c \int \mathcal{R}\left(x, x^{\prime}\right) J\left(x^{\prime}, t\right) d x^{\prime}+S(x, t)
\end{aligned}
$$

where $J$ is the flux in terms of the photon number in units $\mathrm{s}^{-1} \mathrm{~cm}^{-2} . H(t)=\dot{a}(t) / a(t)$ is the Hubble parameter, $a(t)$ being the cosmic factor; $v_{T}=\left(2 k_{B} T / m\right)^{1 / 2}$ is the thermal velocity of hydrogen atoms. The dimensionless frequency $x$ is related to the frequency $\nu$ and the resonant frequency $\nu_{0}$ by $x=\left(\nu-\nu_{0}\right) / \Delta \nu_{D}$, and $\Delta \nu_{D}=\nu_{0} v_{T} / c$ is the Doppler broadening. 
$S(t, x)$ is the source of photons. The parameter $k=\chi / \Delta \nu_{D}$, where $\chi$ is the intensity of the resonant absorption given by $\chi=\pi e^{2} n_{1} f_{12} / m_{e} c$, and $n_{1}$ being the number density of neutral hydrogen $\mathrm{HI}$ at ground state, $f_{12}=0.416$ is the oscillator strength. The cross section of resonant scattering at the line center is

$$
\sigma_{0}=\frac{\pi e^{2}}{m_{e} c} f_{12}\left(\Delta \nu_{D}\right)^{-1} .
$$

In eq.(1),$\phi(x)$ is the profile of the absorption line at the resonant frequency $\nu_{0}$. If the profile is dominated by Doppler broadening, we have

$$
\phi(x)=\frac{1}{\sqrt{\pi}} e^{-x^{2}} .
$$

The redistribution function $\mathcal{R}\left(x, x^{\prime}\right)$ of eq.(11) gives the probability of a photon absorbed at frequency $x^{\prime}$, and isotropically re-emitted at frequency $x$. For coherent scattering, we have (Field 1958; Hummer 1962; Basko 1981)

$$
\mathcal{R}\left(x, x^{\prime}\right)=\frac{1}{2} e^{2 b x^{\prime}+b^{2}} \operatorname{erfc}\left[\max \left(|x+b|,\left|x^{\prime}+b\right|\right)\right],
$$

where the parameter $b=h \nu_{0} / m v_{T} c=2.5 \times 10^{-4}\left(10^{4} / T\right)^{1 / 2}$ is due to the recoil of atoms. It is in the range of $3 \times 10^{-2}-3 \times 10^{-4}$, if the temperature $T$ is in the range of $1 \mathrm{~K}-10^{4} \mathrm{~K}$. The redistribution function is normalized as

$$
\int \mathcal{R}\left(x, x^{\prime}\right) d x=\phi\left(x^{\prime}\right) .
$$

Therefore, we have

$$
k c \int \phi(x) J(x, t) d x=k c \iint \mathcal{R}\left(x, x^{\prime}\right) J\left(x^{\prime}, t\right) d x d x^{\prime} .
$$

It means that the total number of photons absorbed given by the term $k c \phi(x) J(x, t)$ of eq.(1) is equal to the total number of scattered photons. Therefore, with eq.(1), the number of photons is conserved.

\section{2. $\quad$ Rescaling the equations}

We use the new time variable $\tau$ defined as $\tau=c n_{1} \sigma_{0} t$, which is in unit of the mean free flight time of photons at resonant frequency. For the concordance $\Lambda$ CDM model, the number density of hydrogen atoms is $n_{\mathrm{H}}=1.88 \times 10^{-7}\left(\Omega_{b} h^{2} / 0.022\right)(1+z)^{3} \mathrm{~cm}^{-3}$. Therefore, we have

$$
t=0.054 f_{\mathrm{HI}}^{-1}\left(\frac{T}{10^{4}}\right)^{1 / 2}\left(\frac{10}{1+z}\right)^{3}\left(\frac{0.022}{\Omega_{b} h^{2}}\right) \tau \text { yrs },
$$


where $f_{\mathrm{HI}}=n_{1} / n_{\mathrm{H}}$ is the fraction of neutral hydrogen.

We rescale the eq.(1) by the following new variables

$$
J^{\prime}(x, \tau)=[a(t) / a(0)]^{2} J(x, t), \quad S^{\prime}(x, \tau)=[a(t) / a(0)]^{2} S(x, t) .
$$

Thus, eq.(1) becomes

$$
\begin{aligned}
\frac{\partial J^{\prime}(x, \tau)}{\partial \tau}= & -\phi(x) J^{\prime}(x, \tau) \\
& +\int \mathcal{R}\left(x, x^{\prime}\right) J^{\prime}\left(x^{\prime}, \tau\right) d x^{\prime}+\gamma \frac{\partial J^{\prime}}{\partial x}+S^{\prime}(x, \tau)
\end{aligned}
$$

where the parameter $\gamma$ is the so-called Sobolev parameter. $\gamma=\left(H / v_{T} k\right)=\left(8 \pi H / 3 A_{12} \lambda^{3} n_{1}\right)=$ $\left(H m_{e} \nu_{0} / \pi e^{2} n_{1} f_{12}\right)$, where $\lambda$ is the wavelength for Ly $\alpha$ transition. $\gamma^{-1}$ measures the number of scattering during a Hubble time. It actually is the Gunn-Peterson optical depth given by by

$$
\gamma^{-1}=4.9 \times 10^{5} h^{-1} f_{\mathrm{HI}}\left(\frac{0.25}{\Omega_{M}}\right)^{1 / 2}\left(\frac{\Omega_{b} h^{2}}{0.022}\right)\left(\frac{1+z}{10}\right)^{3 / 2} .
$$

Around the first stars, the number $f_{\mathrm{HI}}$ is strongly dependent on time and position (Liu et al. 2007). It is as small as $10^{-6}$ within the ionized sphere, and as high as $\simeq 1$ outside the ionized sphere. Therefore, the parameter $\gamma$ would be in the range from 1 to $10^{-6}$.

The physical meaning of the terms on the right hand side of eq.(9) is clear. The first term is the absorption at frequency $x$, the second term is the re-emission of photons with frequency $x$ by scattering, and the third term describes the redshift of photons. The time scale of a photon moving $\Delta x$ in the frequency space is equal to

$$
\Delta \tau=\gamma^{-1} \Delta x
$$

This actually is due to the Hubble expansion.

Considering eq.(6), eq.(9) gives

$$
\frac{d}{d \tau} \int J^{\prime} d x=\int S^{\prime} d x
$$

This equation shows that the total number of photons $\int J^{\prime} d x$ is dependent only on the sources, regardless of the parameter $b$ of the resonant scattering. Since numerical errors accumulated over a long time evolution could be huge, Eq.(12) is useful to check the reliability of a numerical code. We will use $J$ for $J^{\prime}$ and $S$ for $S^{\prime}$ in sections below. It will not cause confusion. 


\section{Numerical method}

We use the WENO scheme to solve the eq.(9). This algorithm has been given in Roy et al. (2009). Some of the algorithmic details are given in the Appendix. We present a test to show the good performance of our solver below.

Figure 1 plots both the analytical (Field, 1959) and WENO numerical solutions of eq.(9) with parameters $\gamma=0$ and $b=0$. It shows that the numerical solutions can follow the analytical solution in all the time $t$ and the frequency $x$ considered. This result is not trivial if compared with the results of other numerical solvers, such as Meiksin (2006), which shows a large deviation between the analytical and numerical solutions. Therefore, our scheme is more reliable.

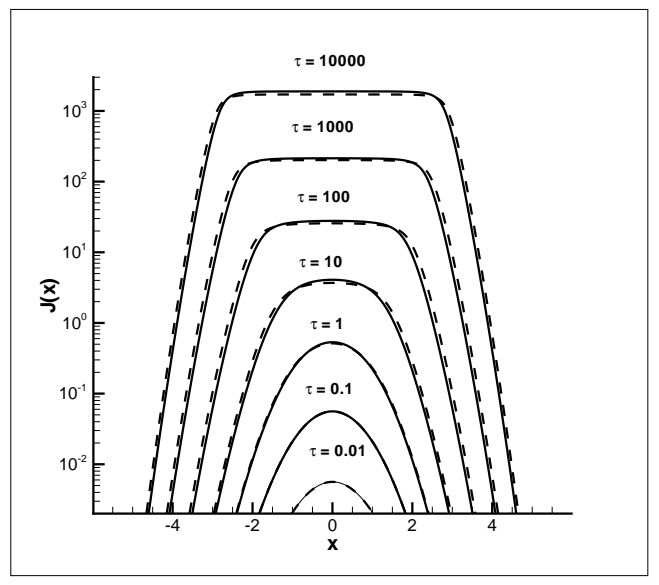

Fig. 1.- Analytical (dashed) and WENO numerical (solid) solutions of eq.(9) with $\gamma=0$ and $b=0$. The source is taken to be $S=\phi(x)$ and initial condition $J(x, 0)=0$.

\section{Wouthuysen-Field coupling in a static background}

To study the effect of atomic recoil, we first solve the time evolution of $J(x, \tau)$ in a static background, i.e. $\gamma=0$. A typical time-dependent result is shown in Figure 2, in which $b=0.03$. The solution of Figure 2 is actually similar to that shown in Figure 1. Figure 1 shows that a flat plateau around $x=0$ is to be formed at $\tau>100$, while Figure 2 shows that $J(x, \tau)$ evolves into a Boltzmann distribution around $x=0$ as

$$
J(x, \tau) \simeq J(0, \tau) e^{-2 b x}=J(0, \tau) e^{-h\left(\nu-\nu_{0}\right) / k T}, \quad|x| \leq x_{l} .
$$

This is the so-called "Planck shape in a region around the initial frequency" (Wouthuysen, 1952). The expression eq.(13) has also been found by Field (1959). We will call this feature 
to be a local Boltzmann distribution. The width $x_{l}$ of the local Boltzmann distribution is numerically defined by the frequency range $|x| \leq x_{l}$, in which the slope $\ln J(x, \tau) / d x$ deviating from $2 b$ is small (see below).

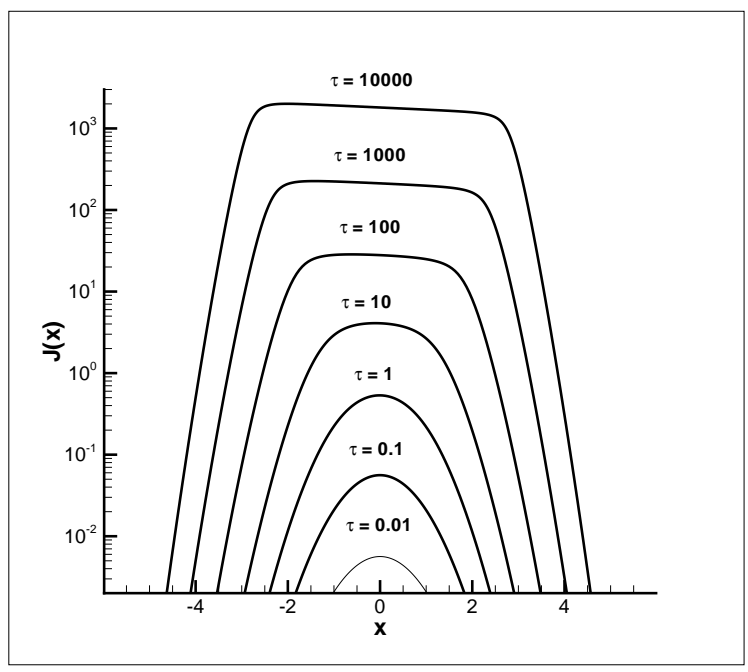

Fig. 2.- WENO numerical solutions of eq.(9) with $\gamma=0$ and $b=0.03$. The source is taken to be $S=\phi(x)$ and initial condition $J(x, 0)=0$.

Figure 3 plots the time-evolution of $J(x, \tau)$ with different parameter $b$, and a zoom-in figure at time $\tau=10^{4}$. The zoom-in figure shows clearly that the integral $\int J(x, \tau) d x$ is $b$-independent. This is consistent with the photon number conservation eq.(12). It shows again that the WENO algorithm is robust. We can also see from the right panel of Figure 3 that all the curves of $\ln J(x, \tau)$ vs. $x$ at $\tau=10^{4}$ and in the range $-2<x<2$ can be approximated as a straight line. That is, the width $x_{l}$ of the local Boltzmann distribution shown in Figure 3 is equaal to about 2, and it is approximately $b$-independent.

The formation and evolution of the local Boltzmann distribution can be quantitatively described by $B(\tau)$ defined as

$$
B(\tau)=2 b /[\ln J(0, \tau)-\ln J(1, \tau)],
$$

where $\ln J(0, \tau)-\ln J(1, \tau)$ is the slope of the straight line $\ln J(x, \tau)$ vs. $x$ for $|x| \leq 1$. For Gaussian source $S(x)=\phi(x)=e^{-x^{2}} / \sqrt{\pi}$, we have $B(0)=2 b$, and $B(\tau)$ approaches $2 b$ at large $\tau$. Figure 4 presents the numerical relation of $B(\tau)$ vs. $\tau$. The slopes $[\log J(0, \tau)-$ $\log J(1, \tau)]$ at $\tau=10^{5}$ are, respectively, 0.0601 for $b=0.030,0.0303$ for $b=0.015,0.0159$ for $b=0.0079$, and 0.0051 for $b=0.0025$. That is, within the frequency range $|x| \leq x_{l}$ and $x_{l}=1$, the relative deviation of the slope $d \ln J(x, \tau) / d x$ from $2 b$ is no larger than $2 \%$. Thus, 
$\tau=10^{5}$ can be considered as the time scale of forming a local Boltzmann distribution within $|x|<x_{l}=1$. For small width $x_{l}<1$, this time scale is lower as $\simeq 10^{4}$. Therefore, the time scale of the onset of W-F coupling with the width $x_{l}$ equal to about Doppler broadening is $10^{4}-10^{5}$.
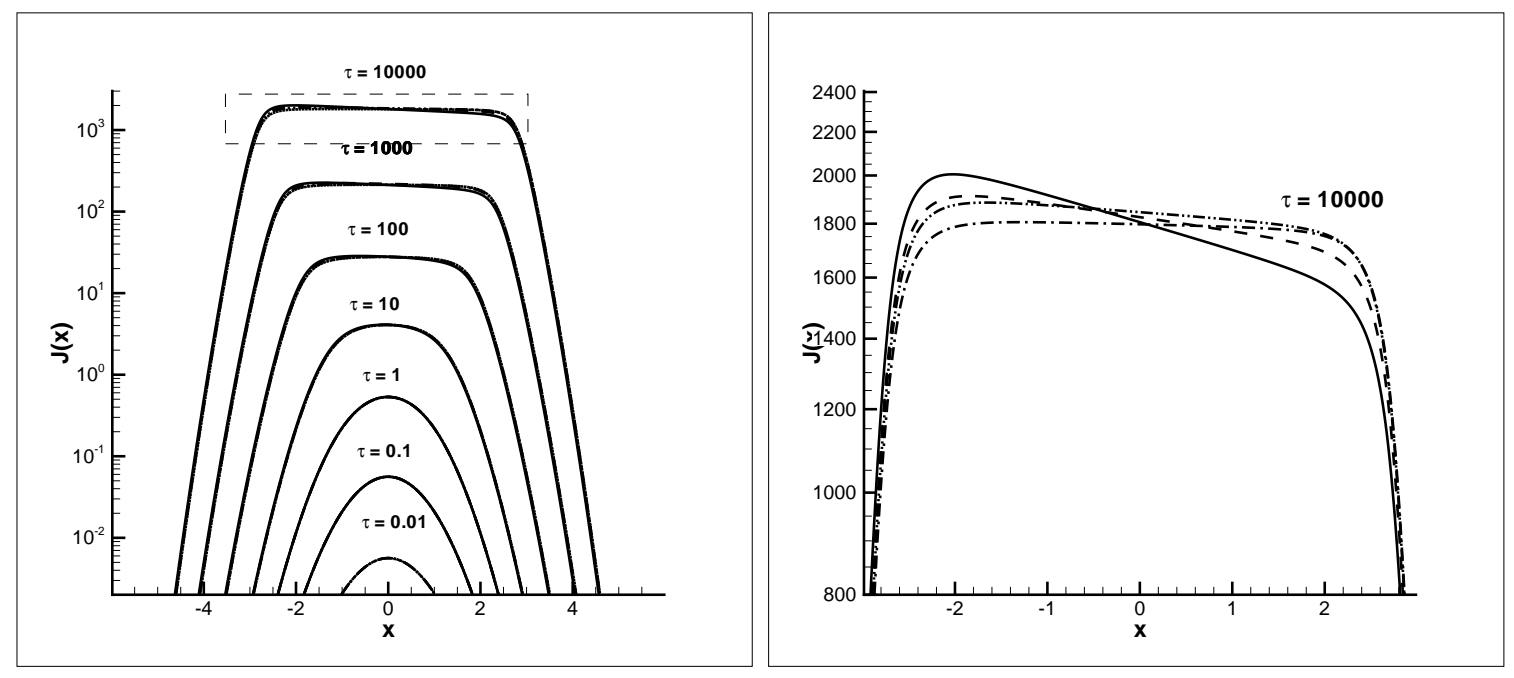

Fig. 3.- WENO numerical solutions of eq.(9) with $\gamma=0$ and $b=0.03$ (solid), 0.015 (dashed), 0.0079 (dot-dot-dashed) and 0.0025 (dot-dashed). The source is taken to be $S=$ $\phi(x)$ and initial condition $J(x, 0)=0$. The right panel is a zoom-in of the dashed square of the left panel.

We can relate the width $x_{l}$ with the mean number of scattering, $N_{c}$, needed to form the local Boltzmann distribution. Although the redistribution function eq.(4) is $b$-dependent, the probability of $x$ photons undergoing a resonant scattering per unit time is $\phi(x)$, which is $b$-independent. Thus, at a given time $\tau$, the mean number $N_{c}$ of resonant scattering of photons within $|x| \leq x_{l}$ approximately is

$$
N_{c} \simeq \tau \frac{1}{x_{l}} \int_{0}^{x_{l}} \phi(x) d x .
$$

Eq.(15) gives the "finite but large number of scattering" for realizing a local Boltzmann distribution within $x \leq x_{l}(\tau)$ (Wouthuysen, 1952). Therefore, the approximate $b$-independence of $x_{l}$ (Figures 3 and 4 ) would imply the $b$-independence of $N_{c}$. 


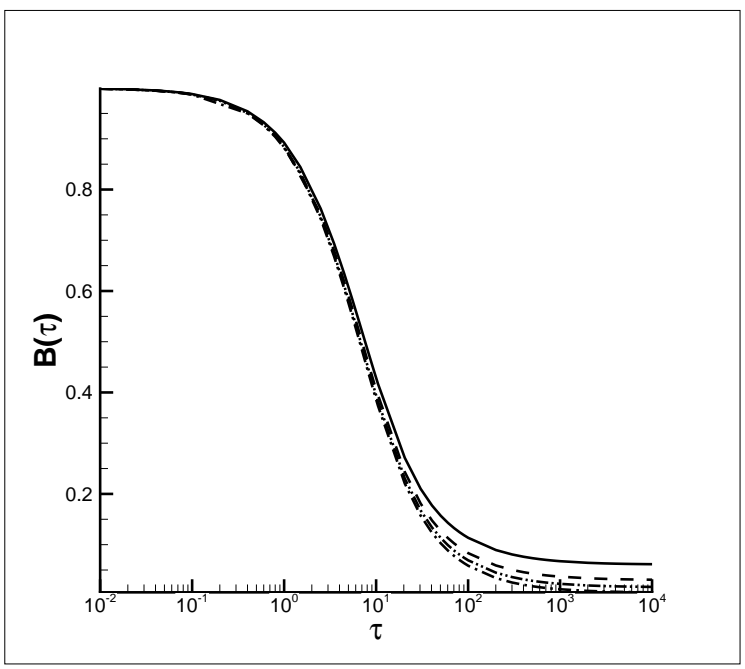

Fig. 4. $-B(\tau)$ vs. $\tau$ for solutions of Figure 3 with $b=0.03$ (solid), 0.015 (dashed), 0.0079 (dot-dot-dashed) and 0.0025 (dot-dashed).

\section{Wouthuysen-Field coupling in an expanding background}

\subsection{Width of the local Boltzmann distribution}

Considering an expanding background, i.e. $\gamma \neq 0$, we solve eq.(9) by the WENO algorithm. Figure 5 plots solutions with the same source $S=\phi(x)$ and parameter $b=0.03$ as in Figure 2, but with $\gamma=10^{-3}$ and $10^{-5}$. Similar to Figure 2, a local Boltzmann distribution has formed when $\tau \geq 10^{3}$ for both $\gamma=10^{-3}$ and $10^{-5}$. The section of the spectrum near $x=0$ becomes $\tau$-independent when $\tau \geq 10^{4}$ for $\gamma=10^{-3}$, and $\tau \geq 10^{6}$ for $\gamma=10^{-5}$. We call this $\tau$-independence to be saturation of the profile $J(x, \tau)$ around resonant frequency. In saturated state, the number of photons redshifted from $\nu>\nu_{0}$ to the local Boltzmann distribution area $\simeq \nu_{0}$ due to Hubble expansion is equal to the number of photons leaving from $\nu_{0}$ to the red wing. Therefore, we see from Figure 5 that once $J$ reaches the saturation state, the boundary on the red wing $(x<0)$ of $J$ is moving to left (red). On the other hand, the boundary on the blue wing $(x>0)$ is almost time-independent.

Unlike in Figure 2, the width $x_{l}$ does not always increase with time. For $\gamma=10^{-3}$ the width stops to increase when $\tau>10^{3}$, and for $\gamma=10^{-5}$, it is stopped at $\tau>10^{5}$. One can find the mean scattering number $N_{c}$ with the similar way as eq.(15). When $\gamma \neq 0$, the time duration of photons staying in the frequency space from $x$ to $x+\Delta x$ roughly is $\Delta x / \gamma$ [eq.(11)]. On the other hand, the mean probability of $|x| \leq x_{l}$ photons being scattered in a unit $\tau$ is $\frac{1}{x_{l}} \int_{0}^{x_{l}} \phi(x) d x$. The larger the $x_{l}$, the less the probability. Thus, all photons within 
$|x|<x_{l}$ averagely undergo $N_{c}$ scattering give by

$$
N_{c} \simeq \frac{1}{\gamma} \int_{0}^{x_{l}} \phi(x) d x
$$

From Figure 5, the maximum width for $\gamma=10^{-3}$ is estimated as $x_{l}=1.9$, corresponding to $N_{c} \simeq 0.5 \times 10^{3}$. While for $\gamma=10^{-5}$, maximum width is $x_{l}=2.8$, and $N_{c} \simeq 5.0 \times 10^{5}$. Once the width $x_{l}$ stops to increse, all quantities in eq.(16), $\gamma, x_{l}$ and $\phi(x)$, are $\tau$-independent. Thus, $N_{c}$ should also be $\tau$-independent.
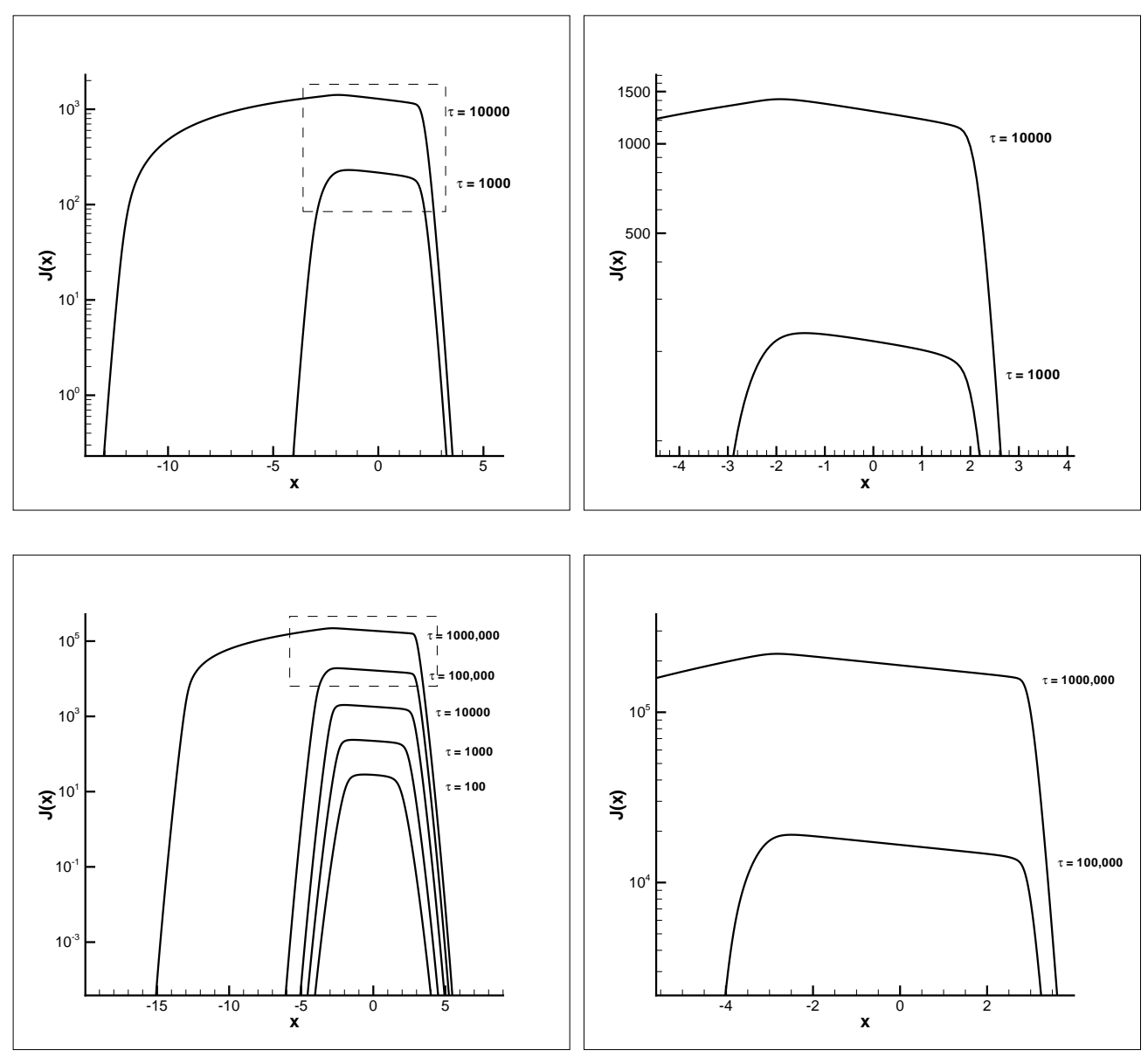

Fig. 5.- WENO numerical solutions of eq.(9) with $b=0.03$ and $\gamma=10^{-3}$ (two top panels) and $\gamma=10^{-5}$ (two bottom panels). The source is taken to be $S=\phi(x)$ and initial condition $J(x, 0)=0$. The right panels are zoom-in of the dashed square of the corresponded left panel.

The $\tau$-independence of the width $x_{l}$ is also shown in Figure 6, in which we still use $\gamma=10^{-3}$, and $J(x, 0)=0$ initially. However, the source is taken to be $S(x)=\phi(x-10)$. 
That is, the source photons have frequency $x=10$, or $\nu=\nu_{0}+10 \Delta \nu_{D}$. The resonant scattering at $x=0\left(\nu=\nu_{0}\right)$ will occur when these photons have redshifted from $\nu$ to $\nu=\nu_{0}$, which takes time of about $\tau=10 / \gamma \simeq 10^{4}$. Figure 6 shows that the whole distribution of $J(x, \tau)$ dramatically evolves with time, but the width of the local Boltzmann distribution around $x=0$ is kept to be $x_{l} \simeq 2$ from $\tau=10^{4}$ to $4 \times 10^{4}$. We also find from our numerical calculations that when $\tau>4 \times 10^{4}$, the intensity of the photon flux around $x=0$ keeps constant, or it is in saturated state.
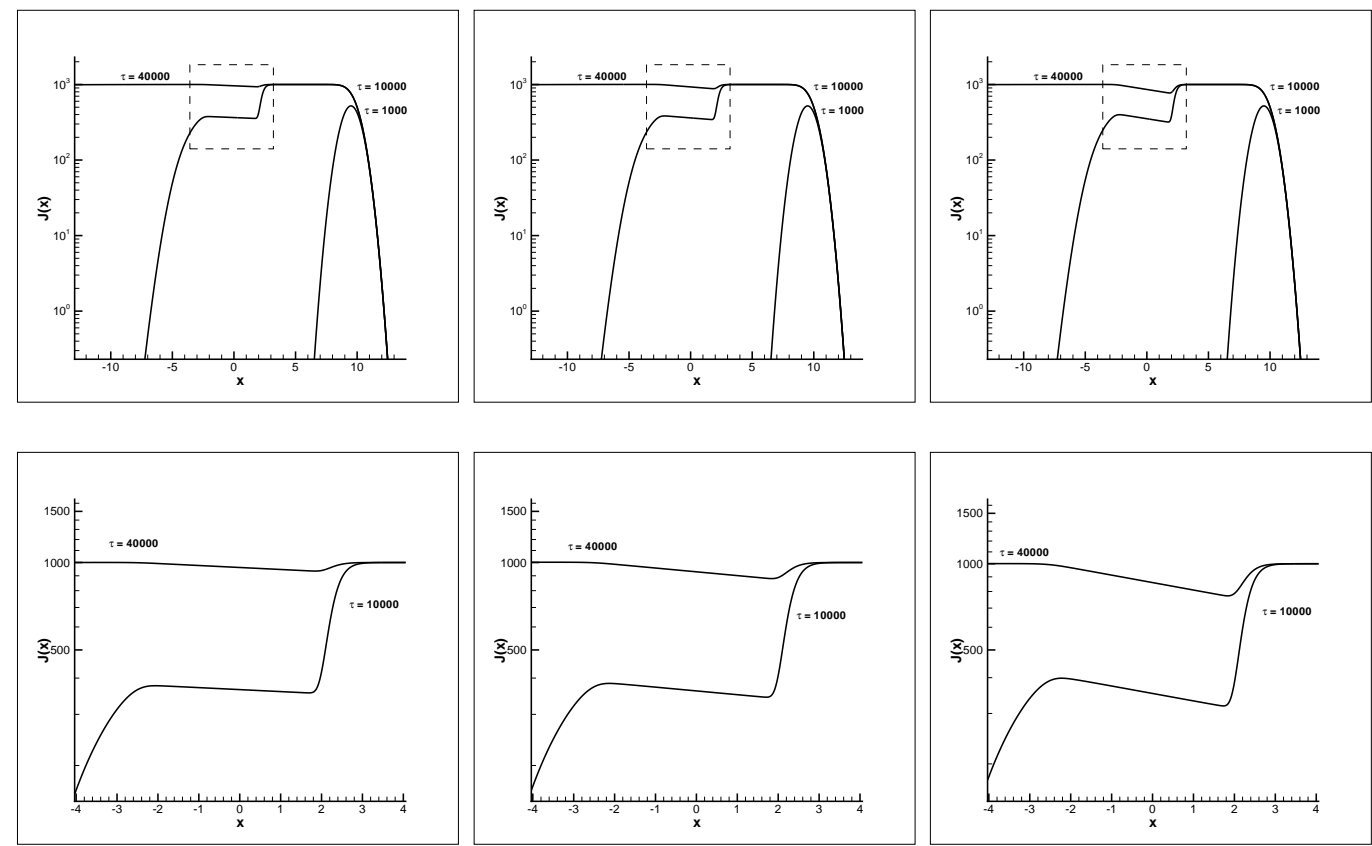

Fig. 6.- WENO numerical solutions of eq.(9) with $\gamma=10^{-3}$ and $b=0.0079$ (left), 0.015 (middle), 0.03 (right). The source is taken to be $S=\phi(x-10)$ and initial condition $J(x, 0)=0$. The bottom panels are the zoom-in of the dashed square of the top panels.

Similar to Figure 3, Figure 6 also shows that the width of the local Boltzmann distribution is approximately $b$-independent. From eq.(16), one can also expect that the width will be smaller for larger $\gamma$. A local Boltzmann distribution can form only if $\gamma^{-1}$ is large enough. This property is shown with Figure 7, in which we use the same photon source $S(x)$ and parameter $b$ as in Figure 6, but we take larger $\gamma$. Figure 7 presents the results of $\gamma=1$ and $10^{-1}$. We see from Figure 7 that in the case of $\gamma=1$, there is no local Boltzmann distribution at any time. The resonant scattering leads only to a valley around $x=0$. It is because the strongest scattering is at $x \simeq 0$, which moves photons with frequency $x \simeq 0$ to $x \neq 0$. However, the redshift lets photon quickly leaving from $x \simeq 0$. They are not 
undergoing enough number of scattering to form a local statistical equilibrium distribution. For $\gamma=10^{-1}$, it seems to show a local Boltzmann distribution, but its width is very small at all time.


Fig. 7.- WENO numerical solutions of eq.(9) with $\gamma=1$ (left) and $\gamma=10^{-1}$ (right). Parameter $b=0.0079$ (dot-dot-dashed), 0.015 (dashed), 0.03 (solid). The source is $S=$ $\phi(x-10)$ and initial condition $J(x, 0)=0$. The bottom panels are the zoom-in of the dashed square of the top panels.

\subsection{Photon source and W-F coupling}

The $\tau$ - and $b$-independencies of the shape and width of the local Boltzmann distribution yield an important conclusion that for given parameters $\gamma$ and $b$, the formation and evolution of the local Boltzmann distribution is independent of the photon sources $S(x, \tau)$. This is because eq.(9) is linear of $J$. Any source $S(x, \tau)$ can always be considered as a superposition of many monochromatic sources around frequency $x=x_{i}$, or $S(x, \tau)=\sum_{i} S_{i} \phi\left(x-x_{i}, \tau\right), S_{i}$ is the intensity of photon source $\phi\left(x-x_{i}, \tau\right)$ with frequency $x_{i} . J(x, \tau)$ can be decomposed into $J(x, \tau)=\sum_{i} J_{i}(x, \tau)$, where $J_{i}(x, \tau)$ is the solution of eq.(9) with the source $i$. Thus, if 
the formation of the local Boltzmann distribution around $x=0$ is independent of $\tau$ and $b$, the superposition $J(x, \tau)=\sum_{i} J_{i}(x, \tau)$ should also show the same local Boltzmann distribution around $x=0$. Although the overall amplitude does depend on the source, the shape around resonant frequency does not.

As an example, Figure 8 presents a solution with the same parameters as in Figure 6, but the source is with continuous spectrum given by

$$
S(x, \tau)= \begin{cases}(10 / x)^{2}, & 10<x<15 \\ 0 & \text { otherwise }\end{cases}
$$

Photons with frequency $x=10$ will arrive earlier at $x=0$ with higher intensity, while those with frequency $x=15$ will arrive at $x=0$ later with lower intensity. The flux $J(x, \tau)$ of Figure 8 has very different shape from Figure 6, while the local Boltzmann distribution at $-2<x<2$ of Figure 8 is exactly the same as that in Figure 6 . Therefore, the W-F coupling is always working regardless the original spectrum of the redshifted photons.
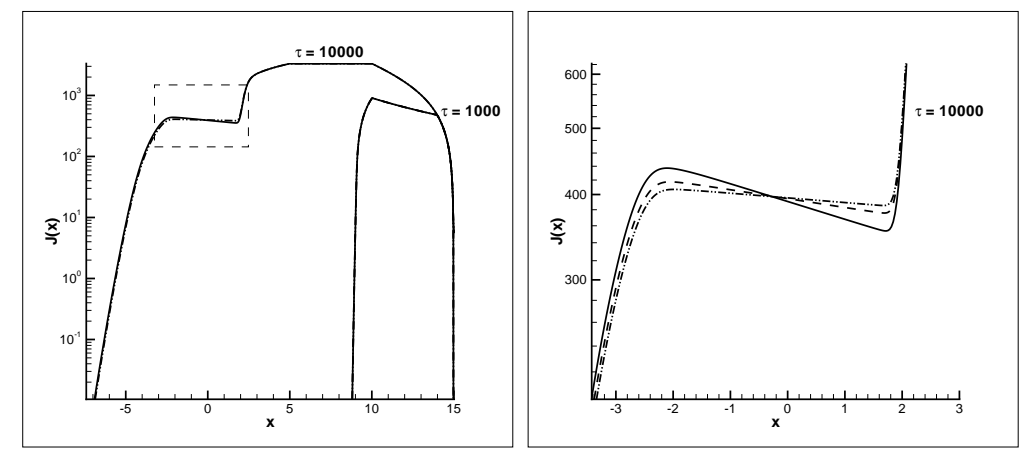

Fig. 8.- WENO numerical solutions of eq.(9) with $\gamma=10^{-3}$ and $b=0.0079$ (dot-dotdashed), 0.015 (dashed), 0.03 (solid). The source is given by eq.(17). The right panel is a zoom-in of the dashed square of left panel.

\subsection{Intensity}

From Figures in $§ 5.1$ and 5.2 , we see that the intensity of photon flux $J$ at the local Boltzmann distribution is strongly dependent on $\gamma$ and $\tau$. Figures 5 and 6 show that at early time $J$ is smaller than its saturation state. For the solution of Figure 6 , the flux in the frequency range of the local Boltzmann distribution is saturated at about $\tau=4 \times 10^{4}$ with saturated flux $J \simeq 10^{4}$, while the intensity at $\tau=10^{4}$ is significantly lower than that of the saturated state. 
Figure 9 is the same as Figure 6, but taking $\gamma=10^{-2}$ and $10^{-4}$. The lower panels of Figure 9 shows once again that the time-evolution of the intensity is about $b$-independent. Figure 9 shows also that for $\gamma=10^{-2}$, the photon flux approaches the saturated state with intensity of $J \simeq 10^{2}$ at $\tau=10^{4}$. While for $\gamma=10^{-4}$, the saturated state has not yet been approached even when intensity $J \simeq 10^{4}$, and $\tau=10^{5}$. Generally, the smaller the $\gamma$, the larger the saturated intensity and the longer the $\tau$ needed to approach its saturated state.
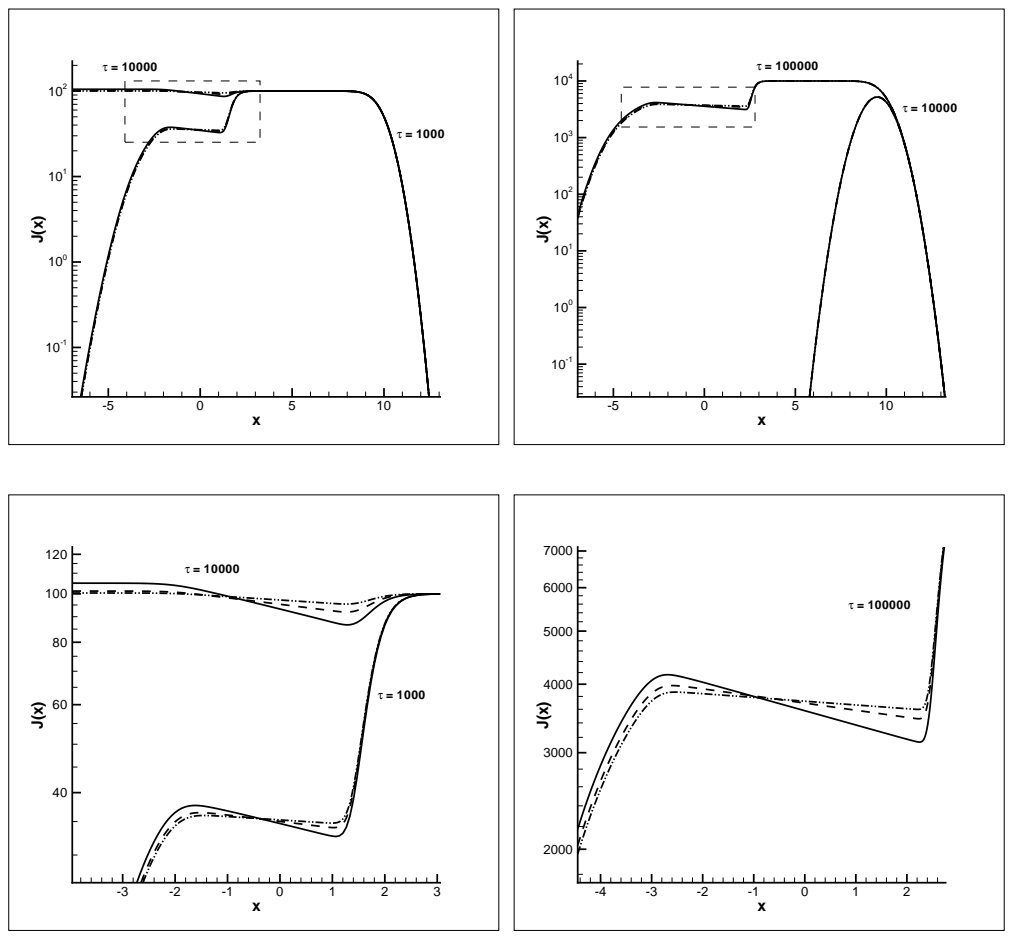

Fig. 9.- WENO numerical solutions of eq.(9) with $\gamma=10^{-2}$ (left) and $10^{-4}$ (right) with source $S(x)=\phi(x-10)$ and $b=0.0079$ (dot-dot-dashed), 0.015 (dashed), 0.03 (solid). The bottom panels are the zoom in of the dashed square of top panels.

\section{Conclusions and discussions}

\subsection{Summary}

The onset of the W-F coupling, or the formation of local Boltzmann distribution is similar to the process of approaching a statistically thermal equilibrium state via collisions or scattering. The particle distribution in the statistical equilibrium is independent of time, initial distribution, and the details of collision. The equilibrium distribution is maintained 
only by the enough collisions among particles. A local Boltzmann distribution is formed once the number of resonant scattering is large enough. Like other statistically thermal equilibrium, the features of the local Boltzmann distribution are independent of time, photon source, initial photon distribution, and etc.

In an expanding universe, photons are moving in the frequency space with "speed" given by the redshift. The formation of the local Boltzmann distribution depends on the competition between the resonant scattering and the redshift. If photons have undergone enough scattering during their path through the frequency space around the resonant frequency, a local Boltzmann distribution will be formed. Otherwise local statistical equilibrium cannot be approached.

In our work, we use the Gaussian profile eq.(3), but not the Voigt profile. Our major results on the formation of local Boltzmann distribution will not be affected by using the Voigt profile if the width $x_{l}$ is not larger than the Doppler thermal broadening. We found that the solution of eq.(9) with rest background is not affected by Voigt profile if the ratio $a$ between the natural and Doppler broadening is equal to $10^{-3}$, which corresponds to $T \simeq 650$ K. For large $a$, or small $T$, the Doppler thermal broadening is small. In this case, the width $x_{l}$, in which the color temperature $T_{c}$ of Ly $\alpha$ photons is locked to the kinetic temperature $T$ of hydrogen atoms, would also be small.

\subsection{Applications to the $21 \mathrm{~cm}$ problem}

A basic problem for the $21 \mathrm{~cm}$ signal from the ionized and heated region around first stars is the conditions on which one can estimate the $21 \mathrm{~cm}$ emission and absorption with the W-F coupling, which forces the internal (spin-)degree of freedom to be determined by the thermal motion of the atoms. In this case, the relative occupation of the two hyperfinestructure components of the ground state depends only upon the shape of the spectrum near the Ly $\alpha$ frequency. Therefore, we need a local Boltzmann distribution of Ly $\alpha$ photon with frequency width equal to or larger than $\left|\nu_{f}-\nu_{0}\right| \geq \nu_{21}=1420 \mathrm{MHz}$, or

$$
x_{f} \geq 0.014\left(\frac{10^{4}}{T}\right)^{1 / 2} .
$$

Thus, from Figure 7 and eq.(10) one can conclude that in regions with $f_{\mathrm{HI}} \leq 10^{-4}$, where $\gamma>10^{-1}$, the W-F coupling will not work. Although electron-hydrogen and proton-hydrogen collisions can be important; the $21 \mathrm{~cm}$ signal will be incredibly small. On the other hand, in the primarily neutral IGM, W-F coupling is very efficient. 
From eqs.(7) and (10), we have

$$
t=0.26 \times 10^{5} h^{-1}\left(\frac{T}{10^{4}}\right)^{1 / 2}\left(\frac{10}{1+z}\right)^{3 / 2}\left(\frac{0.25}{\Omega_{M}}\right)^{1 / 2} \gamma \tau \text { yrs }
$$

We know that the saturation, or time independent solution around resonant frequency, can be used only when the time $\tau$ is larger than the time of photon moving over a frequency space from $-x_{l}$ to $x_{l}$. From Figures 6 and 9, we have $\tau \gamma$ equal to about 10 at the saturation. Therefore, eq.(19) yields that the time independent solution is available only if the time scale of the evolution of the ionized sphere of first stars is larger than about $3 \times 10^{5}$ yrs. This gives a constraint on the $21 \mathrm{~cm}$ emission region, as such regions are very narrow, the time scale of the evolution being comparable to $10^{6} \mathrm{yr}$, or even less (Liu et al. 2007).

Actually we may not need a saturation state. What we used for estimating the $21 \mathrm{~cm}$ signals is the frequency distribution $J(x, \tau)$ to show a Boltzmann-like shape in the central part $|x| \simeq 0$, i.e. the onset of the W-F coupling. The time-scale of the W-F coupling onset is equal to about $10^{3}$ yrs for neutral hydrogen density of the concordance $\Lambda$ CDM model [eq.(7)]. This time scale is much less than that of the evolution of $21 \mathrm{~cm}$ region of first stars. It seems to indicate that we can safely use the W-F coupling in the $21 \mathrm{~cm}$ estimation of first stars.

However, we should mention the effect of the photon intensity. The coupling coefficient between Ly $\alpha$ photons and spin temperature is proportional to the intensity $J$ (e.g. Furlanetto, Oh, \& Briggs 2006). The W-F coupling would generally be suppressed due to the fact that the flux at the resonant frequency $J(x=0)$ is always less than the flux at other frequencies. In a saturated state this suppression is small (Figure 6). However, before approaching the saturated state, the intensity $J$ generally is significantly less than its saturated value. That is, although the local Boltzmann distribution is formed at the time of the order of $\tau \simeq 10^{4}$, the intensity at that time would still be low, and the W-F coupling is not enough to produce the deviation of $T_{s}$ from $T_{\mathrm{CMB}}$. Therefore, it may not be always reasonable to assume that the deviation of $T_{s}$ from $T_{\mathrm{CMB}}$ is mainly due to the W-F coupling if first stars or their emission/absorption regions evolved with time scale equal to or less than Myrs.

The WENO algorithm revealed the time evolution of photons undergoing resonant scattering, whose information is generally lost in the asymptotic solutions, or the timeindependent solution. Although time-independent solutions provide useful guidance, they do not show the conditions for the efficiency of the W-F coupling at different times. The asymptotic solution probably is never reached for short life-time objects. It would be impossible to correctly estimate observable $21 \mathrm{~cm}$ signal from ionized and heated halos of first stars without a correct understanding of the time evolution of the W-F coupling. 
This work is supported by the US NSF under the grants AST-0506734 and AST-0507340.

\section{A. Numerical algorithm}

\section{A.1. Computational domain and computational mesh}

The computational domain in the case of static background is $x \in[-15,15]$. The initial condition is $J(x, 0)=0$ and the boundary condition is $J(x, \tau)=0$ at the boundaries. In the case of expanding background $(\gamma \neq 0)$, the computational domain is bigger, depending on the value of the Sobolev parameter $\gamma$. The domain, denoted as $\left(x_{\text {left }}, x_{\text {right }}\right)$, is chosen such that $J\left(x_{\text {left }}, \tau\right) \approx 0$ and $J\left(x_{\text {right }}, \tau\right) \approx 0$. For example, the domain is taken to be $x \in[-100,15]$ for the case of $\gamma=10^{-3}$.

The computational domain $\left(x_{\text {left }}, x_{\text {right }}\right)$ is discretized into a uniform mesh as following,

$$
x_{i}=i \Delta x, \quad i=-N_{l} \cdots, N_{r},
$$

where $N=N_{l}+N_{r}$ and $\Delta x=\left(x_{\text {right }}-x_{\text {left }}\right) / N$, is the mesh size. We also denote $J_{i}^{n}=$ $J\left(x_{i}, \tau^{n}\right)$, the approximate solution values at $x_{i}$ and the $n^{\text {th }}$ time step, i.e. $\tau^{n}=n \Delta t, \Delta t$ being the numerical time step.

\section{A.2. The WENO algorithm: approximations to spatial derivatives}

To calculate $\frac{\partial J}{\partial x}$, we use the fifth order WENO method (Jiang and Shu, 1996). That is,

$$
\frac{\partial J\left(x_{i}, \tau^{n}\right)}{\partial x} \approx \frac{1}{\Delta x}\left(\hat{h}_{i+1 / 2}-\hat{h}_{i-1 / 2}\right)
$$

where the numerical flux $\hat{h}_{i+1 / 2}$ is obtained by the procedure given below. We use the upwind flux in the fifth order WENO approximation because the wind direction is fixed (negative). First, we denote

$$
h_{i}=J\left(x_{i}, \tau^{n}\right), \quad i=-2,-1, \cdots, N+3
$$

where $n$ is fixed. The numerical flux from the WENO procedure is obtained by

$$
\hat{h}_{i+1 / 2}=\omega_{1} \hat{h}_{i+1 / 2}^{(1)}+\omega_{2} \hat{h}_{i+1 / 2}^{(2)}+\omega_{3} \hat{h}_{i+1 / 2}^{(3)},
$$

where $\hat{h}_{i+1 / 2}^{(m)}$ are the three third order fluxes on three different stencils given by

$$
\hat{h}_{i+1 / 2}^{(1)}=-\frac{1}{6} h_{i-1}+\frac{5}{6} h_{i}+\frac{1}{3} h_{i+1},
$$




$$
\begin{aligned}
& \hat{h}_{i+1 / 2}^{(2)}=\frac{1}{3} h_{i}+\frac{5}{6} h_{i+1}-\frac{1}{6} h_{i+2}, \\
& \hat{h}_{i+1 / 2}^{(3)}=\frac{11}{6} h_{i+1}-\frac{7}{6} h_{i+2}+\frac{1}{3} h_{i+3},
\end{aligned}
$$

and the nonlinear weights $\omega_{m}$ are given by,

$$
\omega_{m}=\frac{\check{\omega}_{m}}{\sum_{l=1}^{3} \check{\omega}_{l}}, \quad \check{\omega}_{l}=\frac{\gamma_{l}}{\left(\epsilon+\beta_{l}\right)^{2}},
$$

where $\epsilon$ is a parameter to avoid the denominator to become zero and is taken as $\epsilon=10^{-8}$. The linear weights $\gamma_{l}$ are given by

$$
\gamma_{1}=\frac{3}{10}, \quad \gamma_{2}=\frac{3}{5}, \quad \gamma_{3}=\frac{1}{10}
$$

and the smoothness indicators $\beta_{l}$ are given by,

$$
\begin{aligned}
& \beta_{1}=\frac{13}{12}\left(h_{i-1}-2 h_{i}+h_{i+1}\right)^{2}+\frac{1}{4}\left(h_{i-1}-4 h_{i}+3 h_{i+1}\right)^{2}, \\
& \beta_{2}=\frac{13}{12}\left(h_{i}-2 h_{i+1}+h_{i+2}\right)^{2}+\frac{1}{4}\left(h_{i}-h_{i+2}\right)^{2}, \\
& \beta_{3}=\frac{13}{12}\left(h_{i+1}-2 h_{i+2}+h_{i+3}\right)^{2}+\frac{1}{4}\left(3 h_{i+1}-4 h_{i+2}+h_{i+3}\right)^{2} .
\end{aligned}
$$

\section{A.3. Numerical integration: an $\mathcal{O}(N)$ algorithm}

We need to numerically integrate $\int \mathcal{R}\left(x, x^{\prime}\right) J\left(x^{\prime}, t\right) d x^{\prime}$, denoted as

$$
I(x)=\frac{1}{2} \int e^{2 b x^{\prime}+b^{2}} \operatorname{erfc}\left(\max \left(|x+b|,\left|x^{\prime}+b\right|\right)\right) J\left(x^{\prime}, t\right) d x^{\prime},
$$

with $\mathcal{R}\left(x, x^{\prime}\right)$ as in eq.(44). To evaluate $I_{m}=I\left(x_{m}\right), \forall m=-N_{l}, \cdots, N_{r}$, we apply the rectangular rule, which is spectrally accurate for smooth functions vanishing at boundaries,

$$
\begin{aligned}
I_{m} & =\frac{1}{2} \int_{x_{\text {left }}}^{x_{\text {right }}} \operatorname{erfc}\left(\max \left(\left|x_{m}+b\right|,\left|x^{\prime}+b\right|\right)\right) e^{2 b x^{\prime}+b^{2}} J\left(x^{\prime}, t\right) d x^{\prime} \\
& \approx \frac{1}{2} \Delta x \sum_{i=-N_{l}}^{N_{r}} \operatorname{erfc}\left(\max \left(\left|x_{m}+b\right|,\left|x_{i}+b\right|\right)\right) e^{2 b x_{i}+b^{2}} J\left(x_{i}, t\right) .
\end{aligned}
$$

Notice that this summation algorithm is very costly as it takes $\mathcal{O}(N)$ operations per $m$, therefore the total procedure has $\mathcal{O}\left(N^{2}\right)$ operations overall. We use a grouping technique, 
described below, so that the overall computational cost can be reduced to $\mathcal{O}(N)$, without changing mathematically the algorithm and its accuracy.

The proposed scheme with order $N$ computational effort is the following. Let $N_{b}=$ floor $\left(\frac{b}{\Delta x}\right)$ and $N_{2 b}=$ floor $\left(\frac{2 b}{\Delta x}\right)$. The integration algorithm is designed for two cases: $m \geq$ $-N_{b}$ and $m<-N_{b}$.

In the case of $m \geq-N_{b}$ or equivalently $x_{m}+b \geq 0$ :

$$
\begin{aligned}
I_{m}= & \frac{1}{2} \Delta x\left(\sum_{i=-N_{l}}^{-m-N_{2 b}-1} \operatorname{erfc}\left(\left|x_{i}+b\right|\right) e^{2 b x_{i}+b^{2}} J\left(x_{i}, t\right)\right. \\
& +\operatorname{erfc}\left(\left|x_{m}+b\right|\right) \sum_{i=-m-N_{2 b}}^{m} e^{2 b x_{i}+b^{2}} J\left(x_{i}, t\right) \\
& \left.+\sum_{i=m+1}^{N_{r}} \operatorname{erfc}\left(\left|x_{i}+b\right|\right) e^{2 b x_{i}+b^{2}} J\left(x_{i}, t\right)\right) \\
\doteq & \frac{1}{2} \Delta x\left(I_{1, m}+\operatorname{erfc}\left(\left|x_{m}+b\right|\right) I_{2, m}+I_{3, m}\right)
\end{aligned}
$$

1. Evaluate $I_{1,-N_{b}}, I_{2,-N_{b}}$ and $I_{3,-N_{b}}$ respectively as

$$
\begin{aligned}
I_{1,-N_{b}} & =\sum_{i=-N_{l}}^{N_{b}-N_{2 b}-1} \operatorname{erfc}\left(\left|x_{i}+b\right|\right) e^{2 b x_{i}+b^{2}} J\left(x_{i}, t\right), \\
I_{2,-N_{b}} & =\sum_{i=N_{b}-N_{2 b}}^{-N_{b}} e^{2 b x_{i}+b^{2}} J\left(x_{i}, t\right), \\
I_{3,-N_{b}} & =\sum_{i=-N_{b}+1}^{N_{r}} \operatorname{erfc}\left(\left|x_{i}+b\right|\right) e^{2 b x_{i}+b^{2}} J\left(x_{i}, t\right),
\end{aligned}
$$

which leads to $\mathcal{O}(N)$ cost.

2. Do $m=-N_{b}+1, N_{r}$

Evaluate $I_{1, m}, I_{2, m}, I_{3, m}$ respectively by

$$
\begin{gathered}
I_{1, m}=I_{1, m-1}-\operatorname{erfc}\left(\left|x_{-m-N_{2 b}}+b\right|\right) e^{2 b x_{-m-N_{2 b}}+b^{2}} J\left(x_{-m-N_{2 b}}, t\right) \\
I_{2, m}=I_{2, m-1}+e^{2 b x_{m}+b^{2}} J\left(x_{m}, t\right)+e^{2 b x_{-m-N_{2 b}}+b^{2}} J\left(x_{-m-N_{2 b}}, t\right) \\
I_{3, m}=I_{3, m-1}-\operatorname{erfc}\left(\left|x_{m}+b\right|\right) e^{2 b x_{m}+b^{2}} J\left(x_{m}, t\right)
\end{gathered}
$$

\section{ENDDO}


To be consistent with the indexes, if $N_{l}-N_{2 b}<N_{r}$ then, we will set $I_{1, m}=0$, for $m=N_{l}-N_{2 b}, N_{r}$. The algorithm leads to $\mathcal{O}(1)$ cost per $m$, therefore $\mathcal{O}(N)$ computation overall.

In the case of $m<-N_{b}$, or equivalently $x_{m}+b<0$ :

$$
\begin{aligned}
I_{m}= & \frac{1}{2} \Delta x\left(\sum_{i=-N_{l}}^{m-1} \operatorname{erfc}\left(\left|x_{i}+b\right|\right) e^{2 b x_{i}+b^{2}} J\left(x_{i}, t\right)\right. \\
& +\operatorname{erfc}\left(\left|x_{m}+b\right|\right) \sum_{i=m}^{-m-N_{2 b}-1} e^{2 b x_{i}+b^{2}} J\left(x_{i}, t\right) \\
& \left.+\sum_{i=-m-N_{2 b}}^{N_{r}} \operatorname{erfc}\left(\left|x_{i}+b\right|\right) e^{2 b x_{i}+b^{2}} J\left(x_{i}, t\right)\right) \\
= & \frac{1}{2} \Delta x\left(I_{1, m}+\operatorname{erfc}\left(\left|x_{m}+b\right|\right) I_{2, m}+I_{3, m}\right)
\end{aligned}
$$

1. Evaluate $I_{1,-N_{b}-1}, I_{2,-N_{b}-1}$ and $I_{3,-N_{b}-1}$ as

$$
\begin{aligned}
I_{1,-N_{b}-1} & =\sum_{i=-N_{l}}^{-N_{b}-2} \operatorname{erfc}\left(\left|x_{i}+b\right|\right) e^{2 b x_{i}+b^{2}} J\left(x_{i}, t\right), \\
I_{2,-N_{b}-1} & =\sum_{i=-N_{b}-1}^{N_{b}-N_{2 b}} e^{2 b x_{i}+b^{2}} J\left(x_{i}, t\right), \\
I_{3,-N_{b}-1} & =\sum_{i=N_{b}+1-N_{2 b}}^{N_{r}} \operatorname{erfc}\left(\left|x_{i}+b\right|\right) e^{2 b x_{i}+b^{2}} J\left(x_{i}, t\right),
\end{aligned}
$$

which leads to $\mathcal{O}(N)$ cost.

2. Do $m=-N_{b}-2,-N_{l}$

Evaluate $I_{1, m}, I_{2, m}, I_{3, m}$ respectively by

$$
\begin{gathered}
I_{1, m}=I_{1, m+1}-\operatorname{erfc}\left(\left|x_{m}+b\right|\right) e^{2 b x_{m}+b^{2}} J\left(x_{m}, t\right) \\
I_{2, m}=I_{2, m+1}+e^{2 b x_{m}+b^{2}} J\left(x_{m}, t\right)+e^{2 b x_{-m-N_{2 b}-1}+b^{2}} J\left(x_{-m-N_{2 b}-1}, t\right) \\
I_{3, m}=I_{3, m+1}-\operatorname{erfc}\left(\left|x_{-m-N_{2 b}-1}+b\right|\right) e^{2 b x_{-m-N_{2 b}-1}+b^{2}} J\left(x_{-m-N_{2 b}-1}, t\right)
\end{gathered}
$$

\section{ENDDO}

To be consistent with the indexes, if $N_{l}-N_{2 b}>N_{r}$, we will set $I_{3, m}=0$, for $m=$ $-N_{2 b}-N_{r},-N_{l}$. Again, the algorithm leads to $\mathcal{O}(1)$ cost per $m$, therefore $\mathcal{O}(N)$ computation overall. 


\section{A.4. Time evolution}

To evolve in time, we use the third-order TVD Runge Kutta time discretization (Shu

\& Osher, 1988). For systems of ODEs $u_{t}=L(u)$, the third order Runge-Kutta method is

$$
\begin{aligned}
u^{(1)} & =u^{n}+\Delta \tau L\left(u^{n}, \tau^{n}\right), \\
u^{(2)} & =\frac{3}{4} u^{n}+\frac{1}{4}\left(u^{(1)}+\Delta \tau L\left(u^{(1)}, \tau^{n}+\Delta \tau\right)\right), \\
u^{n+1} & =\frac{1}{3} u^{n}+\frac{2}{3}\left(u^{(2)}+\Delta \tau L\left(u^{(2)}, \tau^{n}+\frac{1}{2} \Delta \tau\right)\right) .
\end{aligned}
$$

\section{REFERENCES}

Basko, M.M. 1981, Astrophysics, 17, 69

Carrillo, J.A., Gamba, I.M., Majorana, A. \& Shu, C.-W. 2003, Journal of Computational Physics, 184, 498

Cen, R. 2006, ApJ, 648, 47

Chen, X. \& Miralda-Escude, J. 2004, ApJ, 602, 1.

Chuzhoy, L., Alvarez, M. A., \& Shapiro, P. R. 2006, ApJ, 648, L1

Chuzhoy, L. \& Shapiro, P.R. 2006, ApJ, 655, 843

Field, G.B., 1958, Proc. IRE, 46, 240

Field, G.B. 1959, ApJ, 129, 551.

Furlanetto, S.R., Oh, S.P., \& Briggs, F.H. 2006, Physics Reports, 433, 181

Furlanetto, S.R. \& Pritchard, J.R. 2006, MNRAS, 372, 1093

Hirata, C.M. 2006, MNRAS, 367, 259

Hummer, D.G. 1962, MNRAS, 125, 21

Hummer, D.G. \& Rybicki, G.B. 1992, ApJ, 387, 248.

Jiang, G. \& Shu, C.-W. 1996, J. Comput. Phys., 126, 202

Liu, J.-R., Qiu, J.-M., Feng, L.-L., Shu, C.-W. and Fang, L.-Z. 2007, ApJ, 663, 1 
Meiksin, A. 2006, MNRAS, 370, 2025

Qiu, J.-M., Feng, L.-L., Shu, C.-W. \& Fang, L.-Z. 2006, New Astronomy, 12, 1

Qiu, J.-M., Feng, L.-L., Shu, C.-W. \& Fang, L.-Z. 2007, New Astronomy, 12, 398

Qiu, J.-M., Shu, C.-W., Liu, J.-R. \& Fang, L.-Z. 2008, New Astronomy, 13, 1

Roy, I., Qiu J.-M., Shu C.-W., Fang L.-Z., submitted to New Astronomy

Rybicki, G.B. \& Dell'Antonio, I.P. 1994, ApJ, 427, 603

Shu, C.-W. \& Osher, S., 1988, J. Comp. Phys., 77, 439

Wouthuysen, S. A. 1952, AJ, 57, 31 